Article

\title{
Measurement Matrix Analysis and Radiation Improvement of a Metamaterial Aperture Antenna for Coherent Computational Imaging
}

\author{
Na Kou (D), Long Li *, Shuncheng Tian ${ }^{(1)}$ and Yuanchang Li \\ Key Laboratory of High Speed Circuit Design and EMC of Ministry of Education, School of Electronic \\ Engineering, Collaborative Innovation Center of Information Sensing and Understanding, Xidian University, \\ Xi'an 710071, China; kouna02091322@126.com (N.K.); sctian@xidian.edu.cn (S.T.); ycli@stu.xidian.edu.cn (Y.L.) \\ * Correspondence: lilong@mail.xidian.edu.cn; Tel.: +86-29-8820-1157
}

Received: 26 July 2017; Accepted: 7 September 2017; Published: 12 September 2017

\begin{abstract}
A metamaterial aperture antenna (MAA) that generates frequency-diverse radiation field patterns has been introduced in the context of microwave wave imaging to perform compressive image reconstruction. This paper presents a new metamateriapl aperture design, which includes two kinds of metamaterial elements with random distribution. One is a high-Q resonant element whose resonant frequency is agile, and the other one is a low-Q element that has a high radiation efficiency across frequency band. Numerical simulations and measurements show that the radiation efficiency of up to $60 \%$ can be achieved for the MAA and the far-field patterns owns good orthogonality, when using the complementary electric-field-coupled (CELC) element and the complementary Jerusalem cross (CJC) element with a random distribution ratio of 4 to 1 , which could be effectively used to reconstruct the target scattering scene.
\end{abstract}

Keywords: agile frequency response; compressive image reconstruction; metamaterial aperture antenna

\section{Introduction}

In recent years, several physical platforms based on metamaterial aperture antennas (MAAs) have been used to realize computational imaging, as shown in Figure 1. In general, all natural scenes can be compressed on some basis. Scenes can be perfectly reconstructed with significantly fewer measurement modes than the space bandwidth product (SBP) [1-3]. These measurement modes are composed of radiated field patterns of the metamaterial aperture antenna (MAA) at different frequencies. For image reconstruction schemes, which use an arbitrary set of measurement modes, it is essential that the modes are as orthogonal to each other as possible [4] so that the correlation of the modes or field patterns is small [5-7]. Since the measurement modes are indexed by frequency, the low correlation of field patterns can be interpreted as far-field patterns with strong frequency diversity. Hence, to obtain a metamaterial aperture that generates the frequency-agile far-field patterns that are as orthogonal as possible, elements distributed on the aperture must have a strong resonance with a high $Q$-value. Other approaches to frequency-diverse imaging have also been pursued, including multiply scattering structures, such as mode-mixing cavities and dynamic metamaterial apertures [8-10]. Fractal models have been used to design fractal antennas with very special properties: about one-tenth of a wavelength and a pre-fractal geometrical configuration [11] could be used in metamaterial miniaturized technology, which is needed for coherent imaging. The fractional signal, especially its geometrical interpretation, which gives a powerful mathematical tool to model the most advanced concepts of modern physics, could help us to build a simpler mathematical model of the relationship between the metamaterial aperture antenna and its radiation feature, which will improve the speed of full-wave simulation [12]. The initial metamaterial aperture which consists of numerous subwavelength-resonant radiators present a trade-off between the 
$Q$ factor and radiation efficiency (each resonator distributed on aperture is assigned a resonant frequency randomly selected from the bandwidth of operation) [13,14], and most of the power is radiated by the elements closest to the feed, resulting in poor aperture efficiency.

In this paper, the correlation of far-field patterns for metamaterial apertures with different modulations is analyzed, and a trade-off strategy between orthogonality of the far-field patterns and radiation efficiency of the MAA is proposed. The metamaterial aperture is composed of complementary electric-field-coupled elements (CELCs) [15] and complementary Jerusalem cross (CJC) [16] unit cells. The CELC element is strongly resonant with frequency diversity, while the CJC unit cell has high radiation efficiency across the frequency band. By analyzing the filling ratio of the two different elements on the MAA, a relatively low correlation of the far-field patterns and high radiation efficiency is obtained. MAAs with a $20 \times 20$ randomly distributed elements array are theoretically analyzed in the frequency band from 33 to $37 \mathrm{GHz}$. Numerical simulations show that the radiation efficiency of $60 \%$ can be achieved, which yields improved SNRs when using the complementary electric-field-coupled (CELC) element and the CJC element with a random distribution ratio of 4 to 1 on the aperture. A prototype of the MAA with $120 \times 120$ randomly distributed elements whose total size is $250 \mathrm{~mm} \times 250 \mathrm{~mm} \times 0.5 \mathrm{~mm}$ is fabricated to verify the effectiveness of the design and analysis procedure, and to show that the measured far-field patterns across the frequency band from 33 to $37 \mathrm{GHz}$ could be used to effectively reconstruct the target scene with scatterers.
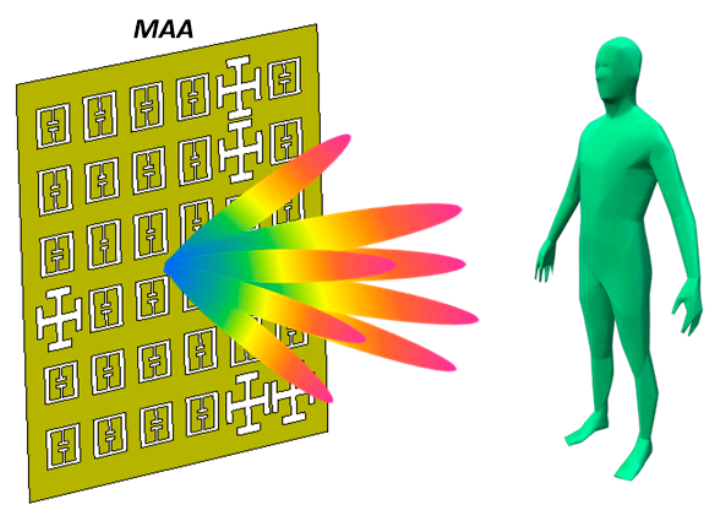

Figure 1. A metamaterial aperture illuminating a human target scene.

\section{The Calculation for the Correlation of Far-Field Patterns of an MAA}

In the context of compressed sensing, the measurement modes can be selected as the radiated far-field patterns of the MAA in a given frequency range. Consider the measurement matrix $H$, which corresponds to the frequency-diverse far-field patterns. $H$ is an $M \times N$ matrix. $M$ represents the quantity of the measurement modes, which is equal to the number of the sampled frequency points. $N$ denotes the far-field pattern pixels at one frequency point, which is the product of the azimuth angles and elevation angles. The matrix reconstruction metric is the average mutual coherence $\mu_{g}$, which is defined as follows [13].

Firstly, with the measurement matrix, the Gram matrix can be obtained $[17,18]$ :

$$
G=H_{n}{ }^{\dagger} H_{n}
$$

where the superscript + represents the conjugate transpose of the matrix, and $H_{n}$ is the measurement matrix $H$ with normalized columns. The average mutual coherence is

$$
\mu_{g}=\frac{\sum_{i \neq j}\left|G_{i j}\right|}{N(N-1)}
$$


As pointed out by Lipworth et al. [13], an increase in the $Q$-factor of the element on the metamaterial aperture causes $\mu_{g}$ to decrease, which indicates that less correlation between measurements represents better orthogonality of the far-field patterns. Here, the frequency quality factor $Q$ can be defined as

$$
Q=\frac{f_{0}}{2 \Delta f}
$$

where $f_{0}$ is the center operating frequency, and $2 \Delta f$ represents the frequency range, which the amplitude response decreases by $3 \mathrm{~dB}$. Usually, when the operating frequency band is given, the center frequency $f_{0}$ is fixed and the frequency interval $\Delta f$ could be changed by selecting different resonant elements with varying frequency agility features. For the strongly resonant element with a high $Q$-value, its frequency selectivity is good, which means the $\Delta f$ or the bandwidth is relatively small. By using this kind of element on the MAA, frequency-agile field patterns with good orthogonality can be obtained. As a result, the target scene can be perfectly reconstructed by utilizing a simple metamaterial aperture antenna instead of mechanical scanning or antenna arrays.

Figure 2 shows the flow chart for calculation procedure of $\mu_{g}$. Firstly, an MAA with randomly distributed elements is designed. At each frequency point, the phase and amplitude responses of the elements on the MAA can be obtained by numerical simulations. Next, the far-field patterns across the frequency band can be calculated using array synthesis technology. Finally, far-field patterns form the measurement matrix and the average mutual coherence $\mu_{g}$ can be obtained. Here we will focus on the theoretical analysis for the variation of $\mu_{g}$ when the $Q$-factor of the resonant elements of the MAA changes.

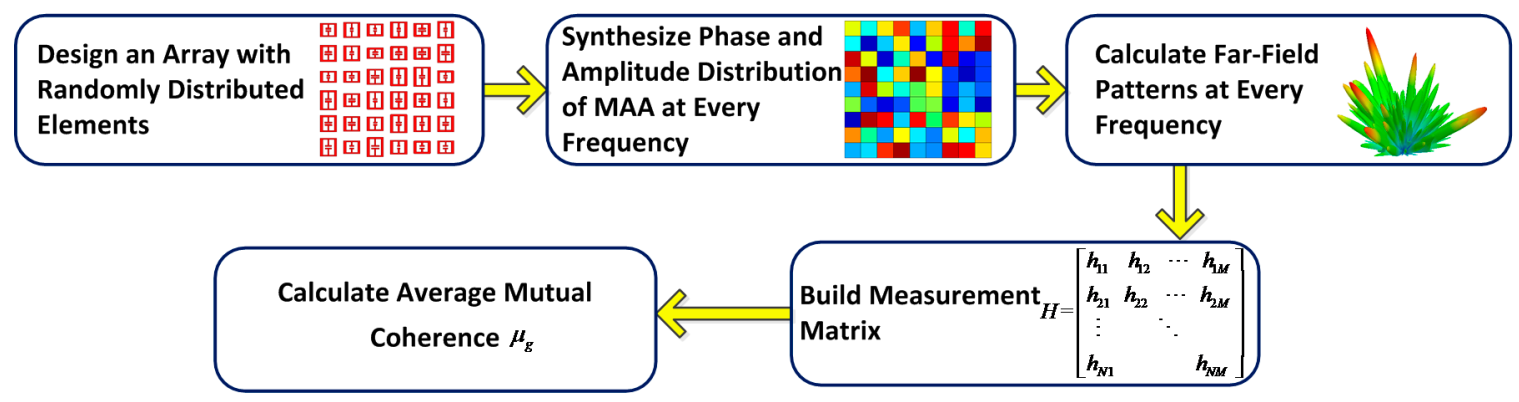

Figure 2. Flow chart of the $\mu_{g}$ analysis procedure.

\section{An Analysis of Average Mutual Coherence $\mu_{g}$}

In order to obtain a set of frequency-diverse far-field patterns whose average mutual coherence $\mu_{g}$ is low, it is essential that different amplitude and phase distributions on one metamaterial aperture are realized at different frequencies from the point of antenna design. However, the strongly resonant elements with frequency-agile responses both on amplitude and phase responses are not realistically achievable. Hence, the phase and amplitude modulations of MAA are performed separately to analyze the average mutual coherence $\mu_{g}$ of its far-field patterns.

For the phase modulation of MAA, the phase distribution on the aperture antenna varies when frequency changes while the amplitude distribution on MAA is uniform. Here, $20 \times 20$ resonant elements with varying phase responses at different frequencies are randomly distributed on the metamaterial aperture antenna, which is analyzed by using array synthesis technology in the frequency band from 33 to $37 \mathrm{GHz}$. The average mutual coherence $\mu_{g}$ of the far-field patterns of the MAA is calculated using elements with varying $Q$-values (or $\Delta f$ ). When the $\Delta f$ of the elements is changed from 0.005 to $0.5 \mathrm{GHz}$, the calculated $\mu_{g}$ varies from 0.02 to 0.13 , as shown in Figure 3 . 


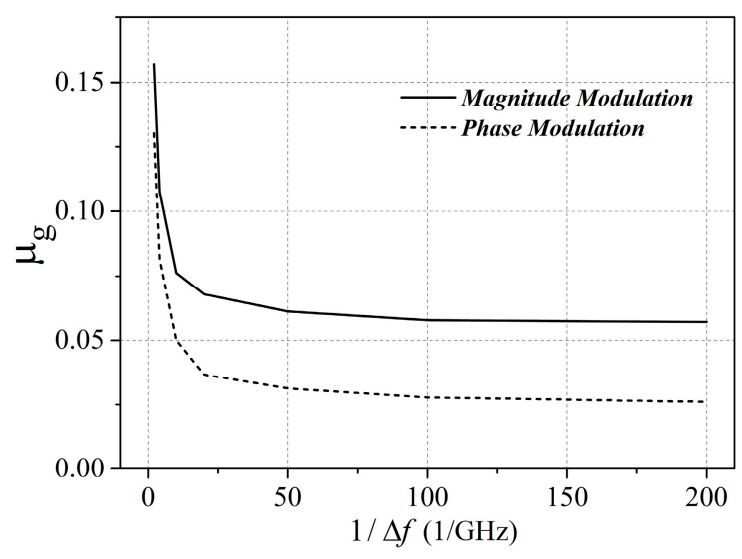

Figure 3. Comparison of the average mutual coherence $\mu_{g}$ between phase and amplitude modulation.

Similarly, for the amplitude modulation of MAA, the amplitude distribution on the aperture is diverse at different frequencies, while the phase distribution on MAA is constant. And $20 \times 20$ resonant elements with diverse amplitude responses in frequency are also distributed on the aperture randomly. By changing $\Delta f$, the average mutual coherence $\mu_{g}$ of the far-field patterns of the MAA is calculated. When the bandwidth $\Delta f$ of the element is changed from 0.005 to $0.5 \mathrm{GHz}$, the calculated $\mu_{g}$ varies from 0.05 to 0.15 . The comparison of $\mu_{g}$ between the phase and amplitude modulation is shown in Figure 3 . It can be seen from Figure 3 that the calculated $\mu_{g}$ of phase modulation is slightly smaller than that of amplitude modulation. When the bandwidth of the element becomes smaller, the average mutual coherence becomes lower. However, the average mutual coherence becomes flat when $\Delta f$ is less than $0.005 \mathrm{GHz}$. It is worth mentioning that the average mutual coherence is irrelevant with respect to the specific location of the center frequency, but it is relative to $\Delta f$. It is common that, at higher operating frequencies, such as the millimeter-wave frequency band, the resonant element often has a larger $\Delta f$ (usually $\geq 0.1 \mathrm{GHz}$ ). Hence, it is much more difficult to design a resonant element with $\Delta f \leq 0.1 \mathrm{GHz}$ at the millimeter-wave band, such as $35 \mathrm{GHz}$.

\section{A Method of Improving the Radiation Efficiency of an MAA}

In general, resonant elements with varying amplitude responses in frequency are realistically achievable. Specifically, different amplitude distributions of the elements on an MAA are represented by the varying radiation efficiencies on the aperture. However, there is a trade-off between the $Q$-factor and the radiation efficiency of these elements [19]. Additionally, an increase in the $Q$-factor of the element on the metamaterial aperture causes $\mu_{g}$ to decrease [13]. Hence, a trade-off between the average mutual coherence $\mu_{g}$ and the radiation efficiency of the MAA must be made.

Usually the MAA is composed of all the strongly resonant elements, such as a CELC [15]. Figure 4 shows the radiation characteristic of a CELC element. The PMC (Perfect Magnetic Conductor) boundary used here is meant to represent the periodic boundary condition that is used to model an infinite metamaterial aperture antenna in practice. It can be seen that, when size parameters $g$ and $l$ decrease, the resonant frequency of the CELC increases and the CELC element has an agile radiation efficiency response across the frequency band. However, the element has a very low radiation efficiency, with a peak value of approximate $50 \%$ and a bandwidth of $\Delta f \approx 0.1 \mathrm{GHz}$. In this case, a low $Q$-factor element can be introduced to increase the radiation efficiency without significantly destroying $\mu_{g}$. The CJC unit cell [16] is adopted, which has a low quality factor but a radiation efficiency that is superior to that of the CELC element, as shown in Figure 5.

In this paper, the improved strategy of the MAA design is a combination of CELC and CJC elements. However, the ratio of the CJC elements distributed on the aperture is crucial, which causes a balance problem between the $\mu_{g}$ and the radiation efficiency of the antenna. To illustrate this, we analyze the performances of the MAA with $20 \times 20$ elements, when the ratio of CJC elements 
changes from 0 to $50 \%$. On the aperture, the CELC elements are used to generate frequency-diverse field patterns. The CJC elements that are used to improve the radiation efficiency are placed on the remaining part of the aperture with a certain ratio. Using a full-wave simulation tool, the radiation efficiency of the CJC element is obtained in the frequency band from 33 to $37 \mathrm{GHz}$, as shown in Figure $5 \mathrm{~b}$. It can be seen that the CJC element has a good radiation ability.

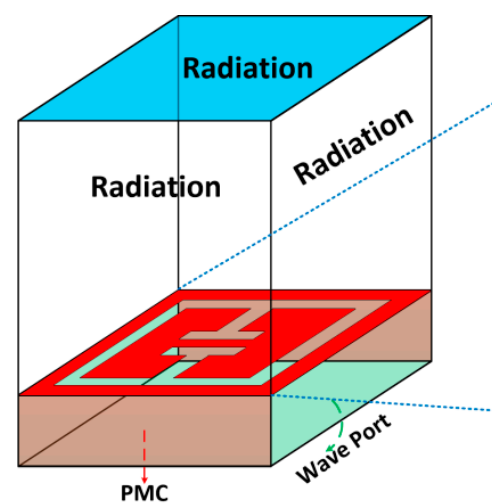

(a)

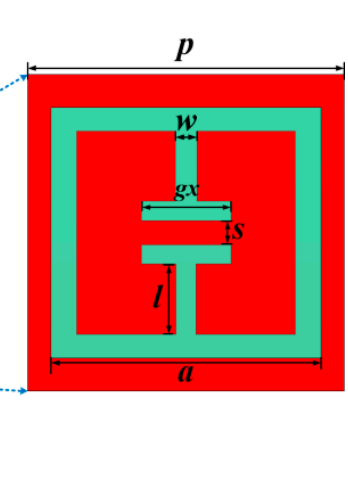

(b)

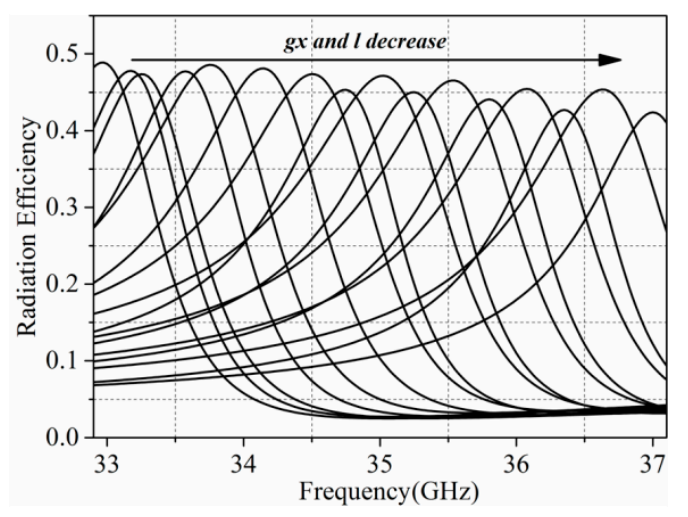

(c)

Figure 4. The complementary electric-field-coupled (CELC) element with a unit cell size of $2 \mathrm{~mm}$ : (a) full-wave simulation model; (b) its top view; (c) the variation in radiation efficiency when $l$ and $g$ change.

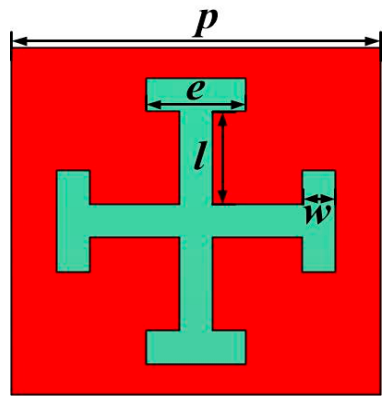

(a)

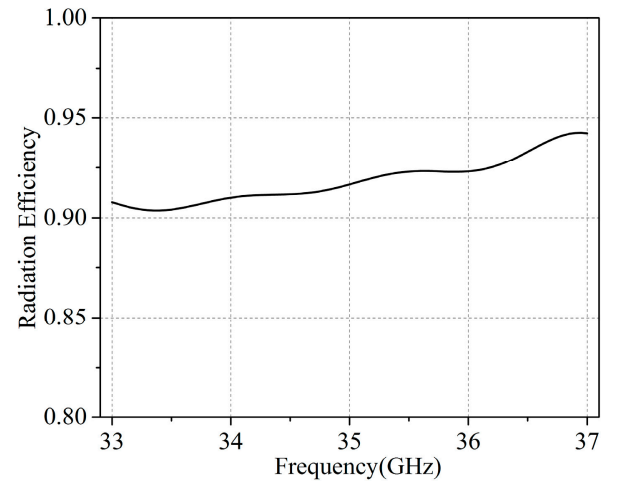

(b)

Figure 5. (a) Top view of complementary Jerusalem cross (CJC) element with a unit cell size of $2 \mathrm{~mm}$ and $(\mathbf{b})$ a radiation efficiency of the element.

When the filling ratio of CJC elements on the MAA is changed from 0 to $50 \%$, as shown in Figure 6, the average mutual coherence $\mu_{g}$ will vary from 0.115 to 0.377 , as shown in Figure $7 \mathrm{a}$. The radiation efficiency of the MAAs with different ratios of CJC elements is shown in Figure 7b. We can see in Figure 7 that the increase in radiation efficiency causes $\mu_{g}$ to increase. As a result, to obtain a relatively high radiation efficiency and a low $\mu_{g}$, the ratio of CJC elements must be selected as $20 \%$ since its radiation efficiency has been improved to around $60 \%$ and its capability of imaging reconstruction approaches that of a metamaterial aperture without CJC elements. 


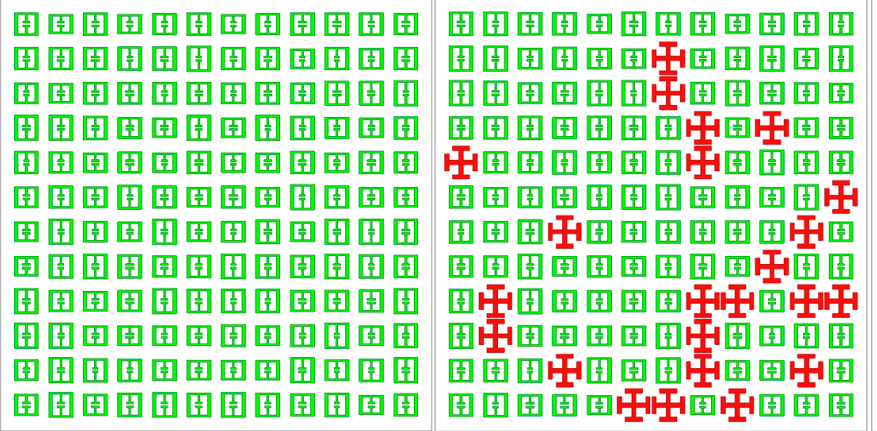

(a) (b)

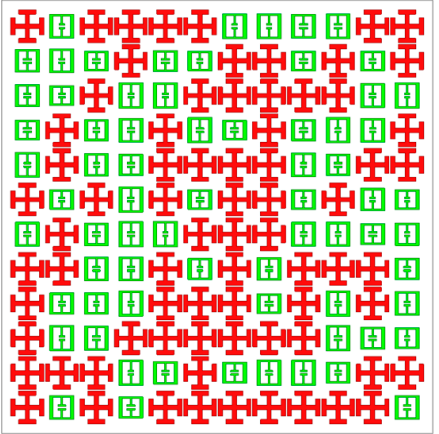

(c)

Figure 6. The schematic of simulated metamaterial aperture antenna (MAA) with different ratios of CJC elements: (a) $0 \%$; (b) 20\%; (c) $50 \%$.

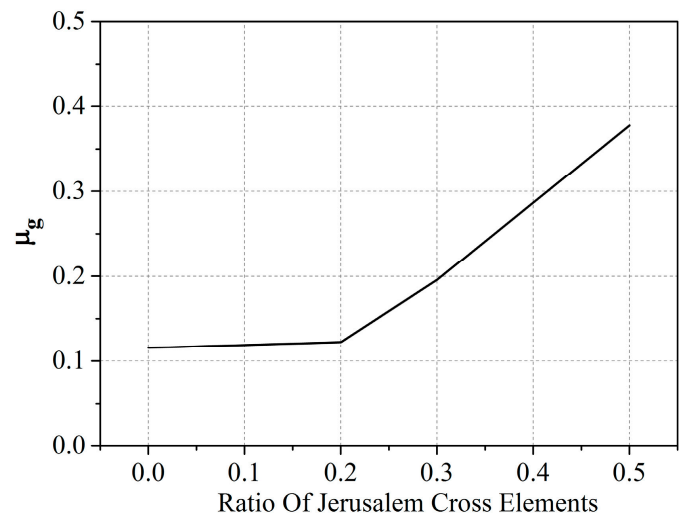

(a)

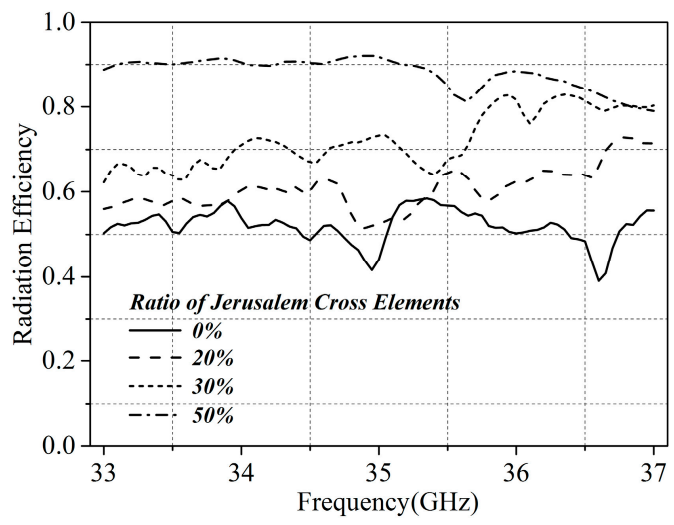

(b)

Figure 7. (a) Calculated average mutual coherence $\mu_{g}$ and (b) the radiation efficiency of different MAAs with different filling ratios of CJC elements.

Thus, the prototype of a metamaterial aperture antenna, with $120 \times 120$ randomly distributed CELC and CJC elements, whose total size is $250 \mathrm{~mm} \times 250 \mathrm{~mm} \times 0.5 \mathrm{~mm}$ was fabricated, measured, and analyzed. The fabricated MAA consisted of $80 \%$ CELC elements and $20 \%$ CJC elements, as shown in Figure 8 b,c. The feed structure of the metamaterial aperture antenna is shown in Figure 8a below. The array consists of four uniform feed structures. The feed structure contains one impedance matching capacitor and four impedance matching inductive pins, and a coaxial feed pin is at the center of the structure. The measurement was performed using near-field planar-scanning techniques and near-to-far-field transformation [20]. The measured far-field patterns across the frequency band from 33 to $37 \mathrm{GHz}$ are shown in Table 1. It can be seen that the designed MAA has frequency-diverse far-field patterns.

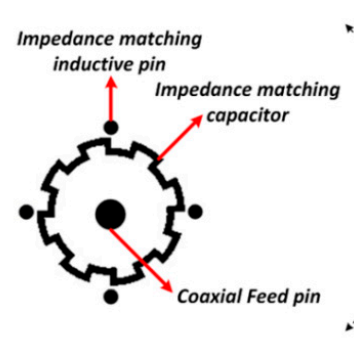

(a)

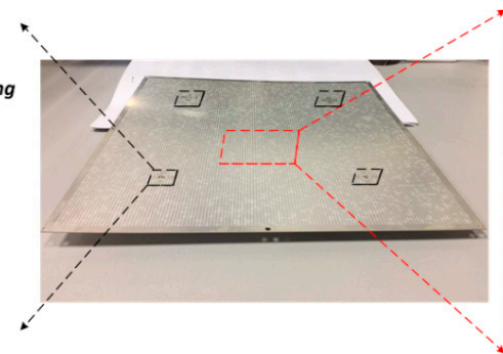

(b)

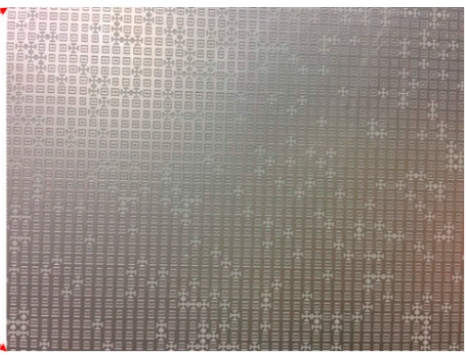

(c)

Figure 8. Geometry of the fabricated MAA: (a) feed structure (the black capacitor part is dielectric, and the remaining part is copper); (b) total view; (c) local view. 
Table 1. Measured field patterns of the metamaterial aperture antenna at different frequencies.

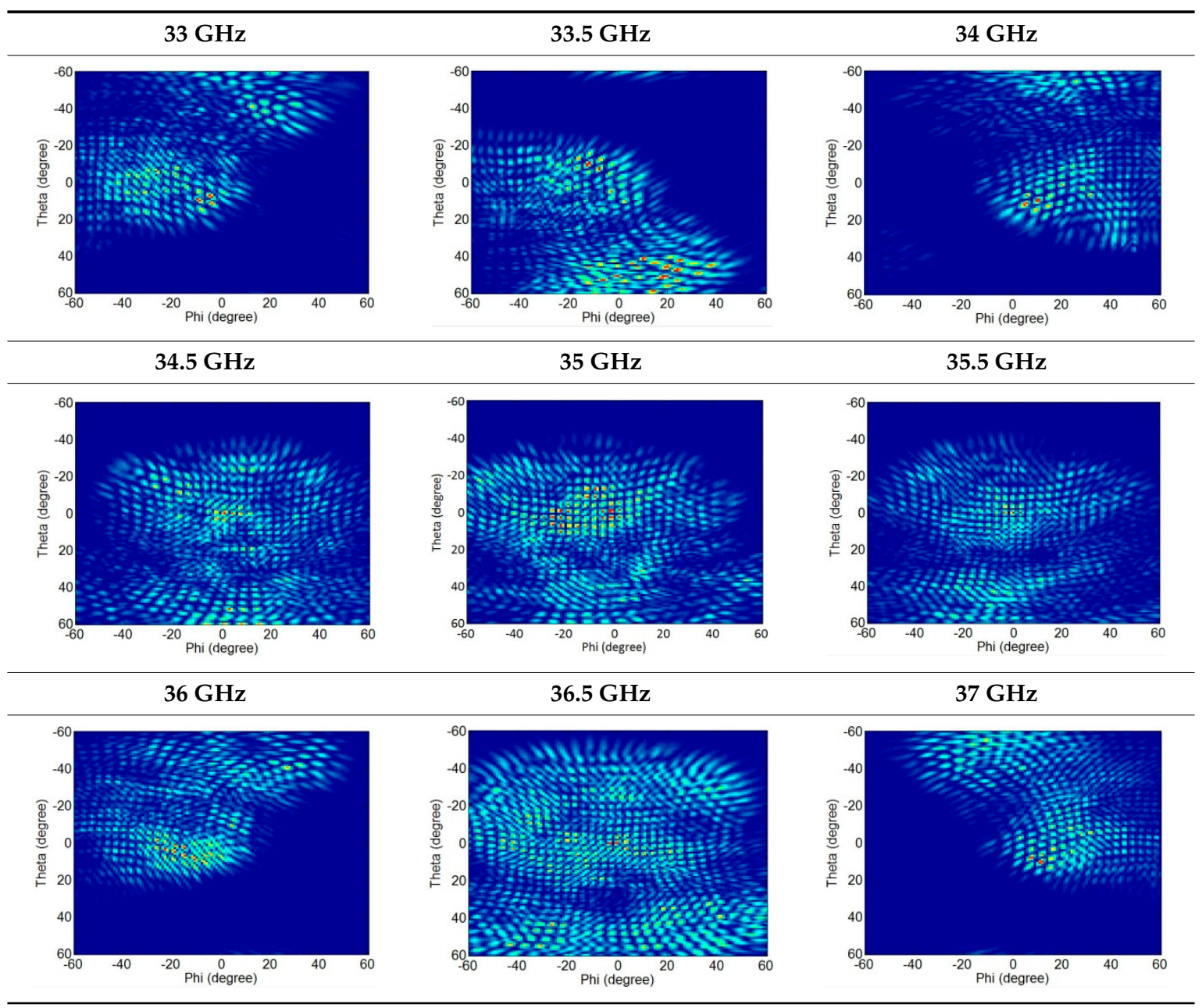

\section{Discussion}

The capability of imaging reconstruction of the fabricated metamaterial aperture antenna was explored based on the measured data. The specific parameters of the MAA's coherent computational imaging system are shown in Table 2. Figure 9a shows the original sparse target scene, which includes two scatterers, and Figure $9 \mathrm{~b}$ shows this scene reconstructed via the pseudo-inverse method. It can be seen in Figure 9 that the designed MAA could be effectively used to reconstruct the target scenes. In conclusion, when using the complementary electric-field-coupled (CELC) element and the complementary Jerusalem cross (CJC) element with a random distribution ratio of 4 to 1 , the radiation efficiency of the MAA can achieve $60 \%$, and the far-field patterns has good orthogonality, which could be effectively used to reconstruct the target scatterers scene.

Table 2. System parameters of the metamaterial aperture antenna.

\begin{tabular}{cc}
\hline Parameters & Value \\
\hline Field of View(Elevation) & $-60-60^{\circ}$ \\
Frequency sampling interval & $0.1 \mathrm{GHz}$ \\
Elevation sampling interval & $1^{\circ}$ \\
Azimuth sampling interval & $1^{\circ}$ \\
\hline
\end{tabular}




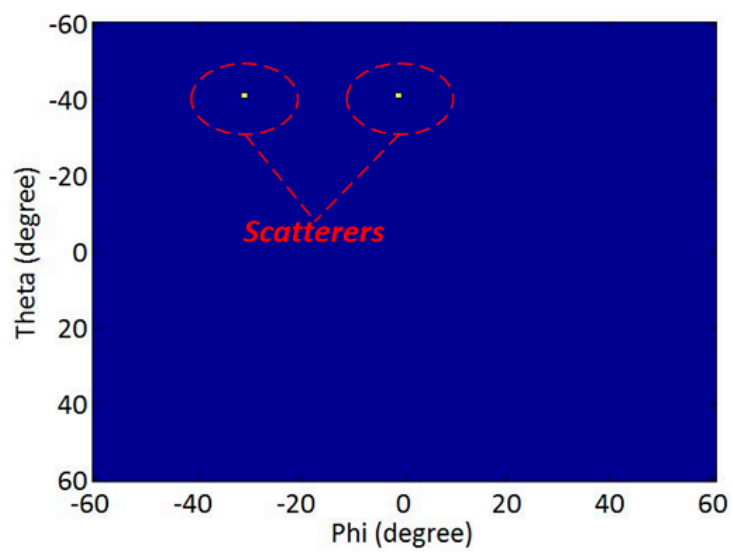

(a)

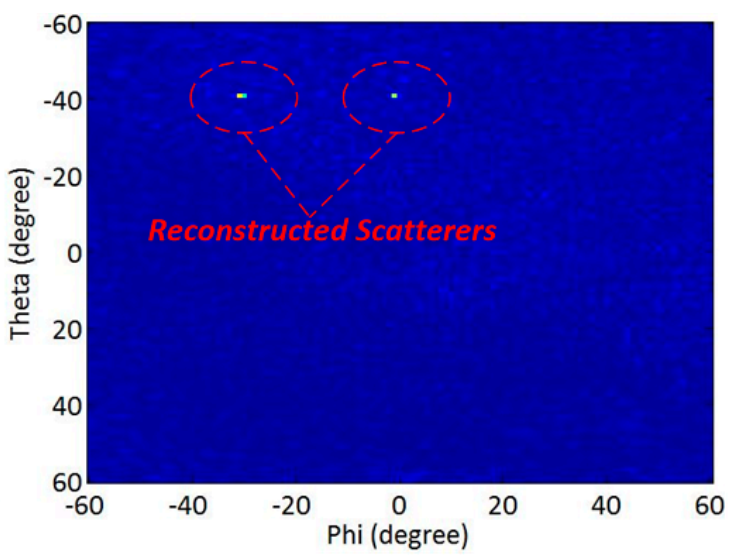

(b)

Figure 9. Target scenes: (a) true target scene consisting of two point scatterers; (b) the same scene reconstructed by the fabricated MAA.

Since the capability of imaging reconstruction of the fabricated metamaterial aperture antenna is explored based on the measured far-field data, the range of the target is in the far-field region. In this case, since the dimension of the fabricated metamaterial aperture antenna is $250 \mathrm{~mm} \times 250 \mathrm{~mm} \times 0.5 \mathrm{~mm}$, and the center frequency is $35 \mathrm{GHz}$, the far-field region is $r \geq(2 \times 0.25 \mathrm{~m} \times 0.25 \mathrm{~m}) / \lambda_{0}=14.5 \mathrm{~m}\left(\lambda_{0}\right.$ is the wavelength at the center frequency in free space). As a result, the true target is assumed to be $15 \mathrm{~m}$ away from the metamaterial aperture antenna.

The capability of imaging reconstruction of the new proposed metamaterial aperture antenna was determined based on simulated targets at the present stage [21]. As for larger targets, we also employed a simulated metal sheet target to validate the capability of imaging reconstruction of the new proposed MAA, as shown in Figure 10 below. In Figure 10, we can see that the measured far-field data could be used to reconstruct the location and shape of the metal sheet more precisely, but not perfectly. Going forward, more practical experiments with a more precise reconstruction of larger targets will be crucial.

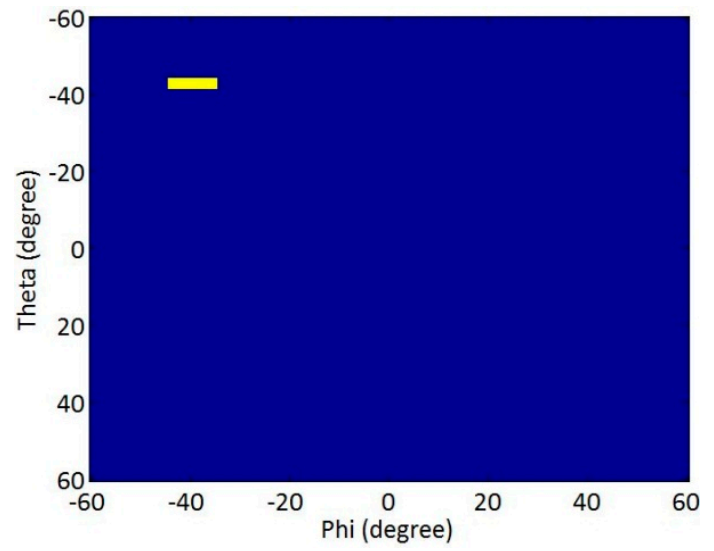

(a)

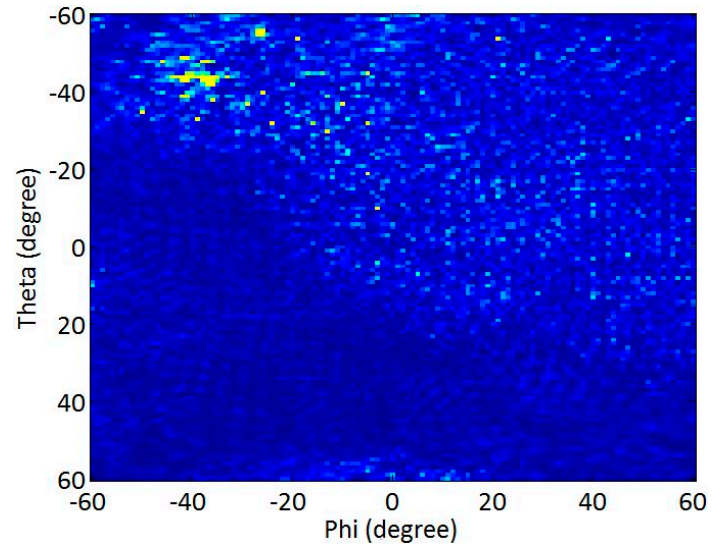

(b)

Figure 10. Target scenes: (a) true target scene consisting of one metal sheet scatterers; (b) the same scene reconstructed by the fabricated MAA.

\section{Conclusions}

A new metamaterial aperture antenna (MAA) composed of two kinds of metamaterial elements with random distribution was proposed, designed, and measured to perform compressive image 
reconstruction. One element has a high $Q$-value with an agile frequency response, and the other element has a relatively low $Q$-factor and has a high radiation efficiency. The orthogonality of the far-field patterns and the improvement in the radiation efficiency of the MAA were theoretically analyzed. Numerical simulations and measurements show that the radiation efficiency of the MAA can achieve $60 \%$, and the far-field patterns have good orthogonality; the MAA can therefore be effectively used to reconstruct scenes with target scatterers when using the complementary electric-field-coupled (CELC) element and the complementary Jerusalem cross (CJC) element with a random distribution ratio of 4 to 1 . Moreover, the proposed MAA can be effectively used for coherent computational imaging.

Acknowledgments: This work was supported by the National Natural Science Foundation of China under Contract No. 51477126, the Technology Explorer and Innovation Research Project, and Fundamental Research Funds for the Central Universities (K5051202051 and SPSZ021409).

Author Contributions: Na Kou conceived the idea of improving the radiation efficiency of the metamaterial aperture antenna and performed the simulations and experiments; Long Li provided the main instructions of the ideas and experiments; Shuncheng Tian and Yuanchang Li designed the feed of the antenna and performed the experiments.

Conflicts of Interest: The authors declare no conflict of interest. The founding sponsors had no role in the design of the study; in the collection, analyses, or interpretation of data; in the writing of the manuscript; or in the decision to publish the results.

\section{References}

1. Hunt, J.; Driscoll, T.; Mrozack, A.; Lipworth, G.; Reynolds, M.; Brady, D.; Smith, D.R. Metamaterial apertures for computational imaging. Science 2013, 339, 310-313. [CrossRef] [PubMed]

2. Brady, D.J.; Choi, K.; Marks, D.L.; Horisaki, R.; Lim, S. Compressive holography. Opt. Express 2009, 17, 13040-13049. [CrossRef] [PubMed]

3. Cull, C.F.; Wikner, D.A.; Mait, J.N.; Mattheiss, M.; Brady, D.J. Millimeter-wave compressive holography. Appl. Opt. 2010, 49, 67-82. [CrossRef] [PubMed]

4. Lohmann, A.W.; Testorf, M.E.; Ojeda-Castañeda, J. The space-bandwidth product, applied to spatial filtering and to holography. Med. J. Aust. 2006, 1, 565.

5. Marks, D.L.; Gollub, J.; Smith, D.R. Spatially resolving antenna arrays using frequency diversity. J. Opt. Soc. Am. A 2016, 33, 899-912. [CrossRef] [PubMed]

6. Sleasman, T.; Imani, M.F.; Gollub, J.N.; Smith, D.R. Microwave Imaging Using a Disordered Cavity with a Dynamically Tunable Impedance Surface. Phys. Rev. Appl. 2016, 6, 054019. [CrossRef]

7. Sleasman, T.; Boyarsk, M.; Imani, M.F.; Gollub, J.N.; Smith, D.R. Design considerations for a dynamic metamaterial aperture for computational imaging at microwave frequencies. J. Opt. Soc. Am. B 2016, $33,1098$. [CrossRef]

8. Fromenteze, T.; Yurduseven, O.; Imani, M.F.; Gollub, J.; Decroze, C.; Carsenat, D.; Smith, D.R. Computational imaging using a mode-mixing cavity at microwave frequencies. Appl. Phys. Lett. 2015, 106, 194104. [CrossRef]

9. Fromenteze, T.; Decroze, C.; Carsenat, D. Waveform Coding for Passive Multiplexing: Application to Microwave Imaging. IEEE Trans. Antennas Propag. 2014, 63, 593-600. [CrossRef]

10. Sleasman, T.; Imani, M.F.; Gollub, J.N.; Smith, D.R. Dynamic metamaterial aperture for microwave imaging. Appl. Phys. Lett. 2015, 107, 204104. [CrossRef]

11. Guariglia, E. Entropy and Fractal Antennas. Entropy 2016, 18, 84. [CrossRef]

12. Guariglia, E. Fractional Derivative of the Riemann Zeta Function. Fract. Dyn. 2015, 21, 357-368.

13. Lipworth, G.; Mrozack, A.; Hunt, J.; Marks, D.L.; Driscoll, T.; Brady, D.; Smith, D.R. Metamaterial microwave holographic imaging system. J. Opt. Soc. Am. A 2013, 30, 1603. [CrossRef] [PubMed]

14. Lipworth, G.; Rose, A.; Yurduseven, O.; Gowda, V.R.; Imani, M.F.; Odabasi, H.; Trofatter, P.; Gollub, J.; Smith, D.R. Comprehensive simulation platform for a metamaterial imaging system. Appl. Opt. 2015, 54, 9343-9353. [CrossRef] [PubMed]

15. Schurig, D.; Mock, J.J.; Smith, D.R. Electric-field-coupled resonators for negative permittivity metamaterials. Appl. Phys. Lett. 2006, 88, 041109. [CrossRef]

16. Arnaud, J.A.; Pelow, F.A. Resonant-Grid Quasi-Optical Diplexers. Electron. Lett. 1973, 9, 589-590. [CrossRef] 
17. Duarte-Carvajalino, J.M.; Sapiro, G. Learning to sense sparse signals: Simultaneous sensing matrix and sparsifying dictionary optimization. IEEE Trans. Image Process. 2009, 18, 1395-1408. [CrossRef] [PubMed]

18. Elad, M. Optimized Projections for Compressed Sensing. IEEE Trans. Signal Process. 2007, 55, 5695-5702. [CrossRef]

19. Chu, L.J. Physical Limitations of Omni-Directional Antennas. J. Appl. Phys. 1948, 19, 1163-1175. [CrossRef]

20. Balanis, C.A. Antenna Theory: Analysis and Design, 3rd ed.; Wiley-Interscience: Hoboken, NJ, USA; Harper \& Row: Bel Air, CA, USA, 2005; pp. 1014-1026.

21. Wu, Z.; Zhang, L.; Liu, H.; Kou, N. Enhancing Microwave Metamaterial Aperture Radar Imaging Performance with Rotation Synthesis. IEEE Sens. J. 2016, 16, 8035-8043. [CrossRef]

(C) 2017 by the authors. Licensee MDPI, Basel, Switzerland. This article is an open access article distributed under the terms and conditions of the Creative Commons Attribution (CC BY) license (http://creativecommons.org/licenses/by/4.0/). 